\title{
Instrumentos de Avaliação de Qualidade de Vida Relacionada à Saúde no Diabetes Melito
}

\author{
RESUMO
}

A avaliação da qualidade de vida (OV) vem se tornando cada vez mais utilizada para medir o impacto geral de doenças na vida dos indivíduos. O diabetes melito (DM) é uma doença crônica associada com morbimortalidade elevada e prejuízo na OV. Em estudos longitudinais, o impacto psicossocial da DM prediz a mortalidade nessa doença. Esta revisão busca descrever e analisar os principais instrumentos de avaliação da OV em pacientes com DM. Foram analisados instrumentos genéricos, como Quality of Well-Being Scale (OWB), The Medical Outcomes Study 36-item Short-Form Health Survey (SF-36) e EuroQol (EQ-5D), e instrumentos específicos, como Diabetes Care Profile (DCP), Diabetes Quality of Life Measure (DOOL), Diabetes Impact Measurement Scales (DIMS), Appraisal of Diabetes Scale (ADS), Audit of Diabetes-Dependent Quality of Life (ADDQoL), Diabetes Health Profile (DHP-1 e DHP-18), Questionnaire on Stress in Patients with Diabetes-Revised (QSD-R), Well-Being Enquiry for Diabetics (WED), Diabetes-Specific Quality-of-life Scale (DSOOLS), Diabetes 39 (D-39) e Problems Areas in Diabetes (PAID). O PAID é o único instrumento traduzido e validado para uso no Brasil. Tanto os instrumentos genéricos quanto os específicos têm vantagens e desvantagens na aferição da OV de pacientes com DM. O uso combinado de instrumentos genéricos (como o SF-36) e específicos (como o PAID) parece ser uma forma consistente de avaliação da QV em pacientes diabéticos no Brasil. O presente artigo revisa os vários instrumentos e enfatiza a necessidade urgente de estudos para validação desses instrumentos em pacientes diabéticos brasileiros. (Arq Bras Endocrinol Metab 2008; 52/6:931-939)

Descritores: Diabetes melito; Qualidade de vida relacionada saúde; Instrumentos de avaliação

\section{ABSTRACT}

\section{Assessment Instruments for a Health-Related Quality of Life in Diabetes} Mellitus.

The assessment of Health-Related Quality of Life (HROoL) has been increasingly used to measure the overall impact of diseases in people's life. Diabetes mellitus (DM) is a chronic disease associated with high morbidity, mortality, and HRQoL impairment in patients. In longitudinal studies, the psychosocial impact of DM predicts mortality. The objective of this review is to describe and to analyze the main instruments used for the HROoL evaluation in patients with DM. Generic instruments such, as the Quality of Well-Being Scale (OWB), Medical Outcomes Study 36-item Short-Form Health Survey (SF-36), EuroOol (EQ-5D) and specific instruments as the Diabetes Care Profile (DCP), Diabetes Quality of Life Measure (DOOL), Diabetes Impact Measurement Scales (DIMS), Appraisal of Diabetes Scale (ADS), Audit of Diabetes-Dependent Quality of Life (ADDQoL), Diabetes Health Profile (DHP-1 and DHP-18), Questionnaire on Stress in Patients with Diabetes-Revised (QSD-R), WellBeing Enquiry goes Diabetics (WED), Diabetes-Specific Quality-of-life Scale revisão

\section{Carlos Clayton torres Aguiar \\ Anya Pimentel G. Fernandes Vieira \\ André FerRer Carvalho \\ ReNAN M. MONTENEGRO-JUNIOR}

Programa de Pós-graduação em Saúde Coletiva da Universidade de Fortaleza (UFC) Serviço de Endocrinologia e Diabetes do Hospital Universitário Walter Cantídio da Faculdade de Medicina da Universidade Federal do Ceará (HUWC-UFC), CE, Brasil (CCTA); Programa de Pós-graduação em Saúde Coletiva da UFC (APGFV); Serviço de Psiquiatria do HUWCUFC; Departamento de Medicina Clínica e Serviço de Psiquiatria da Faculdade de Medicina da UFC (AFC); Serviço de Endocrinologia e Diabetes do HUWC-UFC; Departamento de Saúde Comunitária da Faculdade de Medicina da UFC (RMMJ).

Recebido em 11/1/2008

Aceito em 18/5/2008 
(DSOOLS), Diabetes 39 (D-39) Problems Areas in Diabetes (PAID) were analyzed. PAID is the only translated and validated instrument available in Brazil. The generic and specific instruments have their stregths and shortcomings for evaluation of HRQL in patients with DM. The combined use of both generic (such as the SF-36) and specific (such as the PAID) appears to be a consistent way to evaluate $\mathrm{HRQ}$ oL as a construct in Brazilian patients with DM. The present article reviews a variety of instruments and emphasizes the urgent need for validation studies of such instruments to be used in Brazilian subjects with DM. (Arq Bras Endocrinol Metab 2008; 52/6:931-939)

Keywords: Diabetes mellitus; Health-related quality of life; Instruments for evaluation

\section{INTRODUÇÃO}

O AVAnÇo Tecnológico, desde o início do século XX, criou a expectativa de que a cura das doenças ou os tratamentos eficientes e definitivos seria realidade, porém, apesar dos progressos da medicina, torna-se claro que algumas doenças não são passíveis de cura (1). Entre estas, pode-se mencionar o diabetes melito (DM), patologia com evolução crônica, causando efeitos danosos a longo prazo para o organismo, resultando complicações e prejuízos multidimensionais na vida dos pacientes (2). Pelo número de pessoas afetadas e pelas implicações econômicas envolvidas no seu controle e tratamento, a DM constitui grave problema de saúde pública em todo o mundo (3). Foi consenso, por longo período, a noção de que o impacto das doenças em termos de saúde pública fosse medido pelos índices de mortalidade. Nas patologias crônicas, entretanto, outras perspectivas de avaliação dos resultados estão sendo pesquisadas, levando-se em conta não somente a dimensão médica de redução da morbimortalidade (4). A avaliação da QV do paciente é reconhecida como importante área do conhecimento científico, em razão de o conceito de QV se interpor ao de saúde: satisfação e bem-estar nos âmbitos físico, psíquico, socioeconômico e cultural. A utilização de instrumentos de avaliação da QV permite avaliação mais objetiva e clara do impacto global das doenças crônicas, como o DM na vida dos pacientes. Tal avaliação tem a vantagem de incluir aspectos subjetivos geralmente não abordados por outros critérios de avaliação (5).

O presente ensaio objetiva elaborar breve histórico das definições de QV e revisar instrumentos para avaliação da QV na população diabética.

\section{EVOLUÇÃO HISTÓRICA DA QUALIDADE DE VIDA}

A Organização Mundial da Saúde (OMS), em 1948, definiu saúde como completo estado de bem-estar físico, mental e social e não meramente a ausência de doença (6). A expressão QV, antes tradicionalmente utilizada por filósofos e poetas, passou a ser aplicada por médicos e pesquisadores interessados em avaliar a percepção dos pacientes sobre a doença e o seu respectivo tratamento (7). O número de pesquisas sobre o tema vem em crescimento desde a década de 1970 (8) pela utilização dos resultados obtidos na QV relacionada à saúde (QVRS), não só para descrever o fenômeno saúde/doença, mas também para ajudar os clínicos e gestores em saúde a mais bem avaliarem o impacto das terapêuticas e políticas de saúde (9).

$\mathrm{O}$ crescimento do número de pesquisas e artigos na área fez que, em 1977, a dicção "qualidade de vida" fosse criada como expressão-chave no Medical Subject Heading (MeSH) da US National Libray of Medicine (MedLine). O interesse pela QV nos últimos tempos pode ser demonstrado pelo número crescente de artigos. Em pesquisa realizada no MedLine, foram encontradas 140 referências para o período entre 1966 e 1974, 4.605 referências entre 1966 e 1986, e mais de 62 mil referências nos últimos dez anos sobre a QV.

Inicialmente na década de 1970, os pesquisadores se concentraram na construção e no desenvolvimento de instrumentos que buscavam quantificar a QV (10). Em 1973, todavia, o Quality of Well Being Scale (QWB) ampliou as funções dos instrumentos de avaliação, incorporando julgamentos de valor na avaliação do status de saúde ou nos resultados do tratamento $(11,12)$, e o 
Sickness Impact Profile (SIP) (13) foi elaborado para ser utilizado na avaliação dos cuidados de saúde (bealth care). Com o contínuo e crescente interesse na área, inúmeros instrumentos foram instituídos na década seguinte.

É interessante saber que os instrumentos de avaliação de QV geralmente são construídos em países de língua inglesa, para, posteriormente, serem traduzidos e aplicados em diferentes culturas. A utilização desses questionários em culturas diversas recebe muitas críticas em decorrência das peculiaridades de determinadas populações (14). Outra crítica, por exemplo, é que muitos desses questionários não foram submetidos a estudos adequados de validação antes de serem utilizados em pesquisas epidemiológicas. A carência de ferramentas que avaliassem QV dentro da perspectiva transcultural motivou a OMS a elaborar o WHOQOL-100, no início da década de 1990 (15). Esse processo iniciou-se com a criação do grupo multicêntrico de estudo sobre QV, que a definiu como: "a percepção do indivíduo de sua posição na vida, no contexto da cultura e sistemas de valores nos quais vive e em relação aos seus objetivos, expectativas, padrões e preocupações" (16), entendimento que continua hoje sendo utilizado por alguns pesquisadores da área.

\section{DIABETES MELITO E QUALIDADE DE VIDA}

Até o momento, os determinantes de QV do paciente diabético continuam indefinidos. Apesar disso, existe consenso: o DM impacta na QV (17). Estudos demonstram que pacientes com DM têm nível de QV menor do que os pacientes sem esta enfermidade (18). Neste possível impacto negativo da DM sobre a QV, os aspectos que estão envolvidos ainda não são claramente conhecidos (19). Sabe-se, contudo, que número enorme de variáveis (tipo de DM, uso de insulina, idade, complicações, nível social, fatores psicológicos, etnias, educação, conhecimento sobre a doença, tipo de assistência etc.) pode influenciar a QV em pacientes diabéticos.

Existem vários estudos com dados conflitantes em relação à influência do uso de insulina na QV. O uso da insulinoterapia em algumas pesquisas está associado a valores baixos de QV $(20,21)$; em outras pesquisas, o uso da insulina pode melhorar a QV (22-24), ou até mesmo não influenciá-la (25). A avaliação de QV em pacientes diabéticos é bem mais complexa e inter-relacionada e a insulinoterapia pode levar ao melhor controle glicêmico, mas também pode aumentar o risco de hipoglicemia em pacientes com DM.
A presença de complicações clínicas decorrentes da DM tem impacto potencialmente significante na QV, visto que, quanto maior o número de complicações, pior é a QV do paciente (26). O aumento da prevalência da obesidade e sua associação com a DM tipo 2 (DM2) é cada vez mais pesquisada, incluindo a elaboração de instrumento específico de avaliação de QV (27). Os pacientes obesos com DM2 apresentam melhora na QV após perda de peso (28).

Dessa forma, a avaliação da QV é constantemente incorporada a estudos clínico-epidemiológicos envolvendo pacientes com DM. Tal desfecho tem o potencial de refletir, de maneira mais global, o estado de saúde e o bem-estar desse grupo heterogêneo de pacientes. A escolha do instrumento que deve ser utilizado para avaliar a QV é fator importante.

\section{Instrumentos de avaliação de qualidade de vida}

As escalas de avaliação de QV podem ser divididas em genéricas e específicas.

As escalas genéricas de avaliação de QV são multidimensionais e foram desenvolvidas com o objetivo de avaliar o impacto causado por uma doença, avaliando vários aspectos (capacidade funcional, aspectos físicos, dor, estado geral de saúde, vitalidade, aspectos sociais, aspectos emocionais e saúde mental). Estas escalas também podem ser utilizadas para avaliar a eficácia de políticas e programas de saúde, como a alocação de recursos a serem utilizados, ou para comparar duas enfermidades distintas (paciente com DM comparado com pacientes com artrite reumatóide). O QWB e o SIP são exemplos destas escalas.

As escalas específicas, geralmente, também são multidimensionais e avaliam ainda a percepção geral da QV, embora a ênfase habitualmente seja sobre sintomas, incapacidades ou limitações relacionados a determinada enfermidade. Elas têm a vantagem de analisar mais detalhadamente as alterações na QV em determinadas situações (patologias). Avaliam de maneira específica e profunda aspectos de $\mathrm{QV}$, como dor, capacidade funcional e status emocional. Alguns exemplos de questionários específicos utilizados em outras doenças, já validados para uso no Brasil e utilizados são: Kidney Disease and Quality-of-Life Short-Form (KDQOLSFTM), específico para doença renal crônica terminal, auto-aplicável, com 80 itens (29-31) e o Acromegaly Quality of Life Questionnaire (ACROQoL), questionário unidimensional que contém 22 itens divididos em 
dois domínios: um que avalia os aspectos físicos (8 itens) e outro que afere os aspectos psicológicos de pacientes com acromegalia (14 itens). Este último também é dividido em duas subdivisões: uma delas avalia a aparência física e a outra mede o impacto da doença nas relações pessoais do paciente ( 7 itens cada) $(32,33)$.

Um aspecto importante a ser considerado em relação aos instrumentos é a sua confiabilidade, que se refere ao quanto uma medida pode ser reaplicada repetidamente nos mesmos indivíduos, obtendo-se resultados semelhantes (em diferentes intervalos de tempo e por variados entrevistadores). Dessa forma, avaliam-se a quantidade de erros aleatórios, como também os aspectos sistemáticos próprios do instrumento. Para se comprovar isso, avalia-se um mesmo indivíduo que não foi alvo de alteração clínica em dois momentos diferentes e espera-se que o resultado não seja alterado, isto é, que o resultado permaneça o mesmo, portanto, a confiabilidade é medida por sua reprodutibilidade teste/ reteste, além da reprodutibilidade do interobservador. Estatisticamente, uma das formas de se avaliar é o coeficiente alfa de Cronbach (34), que deve apresentar índice de confiabilidade (consistência interna) de, no mínimo, 0,7, quando o instrumento é pretendido para uso em grupos, e de 0,9 quando se pretende utilizar em indivíduos.

\section{Instrumentos genéricos utilizados no diabetes melito}

O EuroQol (EQ-5D) (35) é instrumento genérico, multidimensional, que avalia o estado de saúde. O EQ-5D enseja cinco domínios: mobilidade, cuidados próprios, atividade habitual, dor/desconforto e ansiedade/depressão. Para cada item, existe gradação de 1 , 2 e 3 (sem problemas, algum problema e problema grave, respectivamente) (36). Ele também inclui escala analógica visual (EAV) em que o paciente gradua seu estado geral de saúde de 0 (pior imaginável) a 100 (melhor imaginável).

O QWB (37) é instrumento que afere o bem-estar em indivíduos com base em três domínios: mobilidade, atividade física e atividade social. Além disso, inclui uma lista de sintomas e problemas que podem interferir na QV. Sua pontuação varia de 0 a 1 . Faz a avaliação referente aos seis últimos dias. O QWB é relativamente longo e complexo e requer entrevistador treinado. Em 1996, foi lançada a versão auto-respondida (Quality of Well-Being Scale Self-Administered) (38).
Em 1992, o The Medical Outcomes Study 36-item Short-Form Health Survey (SF-36) foi elaborado como instrumento genérico (39). O SF-36 é um questionário multidimensional de fácil aplicação e entendimento. É composto por 11 questões e 36 itens, em oito domínios: capacidade funcional ( 10 itens), aspectos físicos (4 itens), dor ( 2 itens), estado geral de saúde (5 itens), vitalidade (4 itens), aspectos sociais ( 2 itens), aspectos emocionais ( 3 itens) e saúde mental ( 5 itens). Para cada domínio o valor varia de 0 a 100 , em que 0 corresponde ao pior e 100 ao melhor estado de saúde. O questionário já foi traduzido para o português e validado no Brasil (7). Em estudo sobre a produção científica acerca da temática QV, produzida pelas universidades públicas do Estado de São Paulo, o SF-36 foi o instrumento mais utilizado, estando presente em 18 estudos, representando $33,9 \%$ do total estudado. Em oito estudos, foi o único instrumento a ser utilizado; já nos outros dez experimentos esteve associado a instrumento do tipo específico (40).

\section{Instrumentos específicos para o diabetes melito}

O Diabetes Care Profile (DCP) (41) avalia fatores psicológicos e sociais associados à doença e ao tratamento, mediante 234 questões auto-administradas. O DCP pode ser aplicado em qualquer raça ou faixa etária. Possui consistência interna (alfa de Cronbach) variando entre 0,60 até $0,95(42)$.

O Diabetes Quality of Life Measure (DQOL) (43), com 46 perguntas, foi criado para ser aplicado em um estudo clínico, multicêntrico, randomizado, com o objetivo de avaliar os efeitos do tratamento intensivo em pacientes com DM tipo I (DMI). Deve ser utilizado para pacientes com DM em uso de insulina (44). Seu emprego foi aprovado para adultos e adolescentes, mas não foi adequado para pacientes com DM2, nem para idosos (45). Sua confiabilidade obteve consistência interna (alfa de Cronbach) variando entre 0,67 até 0,92 (42).

O Diabetes-Specific Quality-of-life Scale (DSQOLS) (46) tem 64 questões em três domínios (objetivos individuais do tratamento, satisfação com o sucesso do tratamento e sofrimento relacionado ao DM). A idade do paciente e a duração da doença estiveram significantemente associadas às queixas físicas e à flexibilidade de tempo para o lazer. Foi desenvolvido para ser utilizado em pacientes com DMl, e tem consistência interna (alfa de Cronbach) variando entre 0,70 até 0,88 (42). 
O Diabetes Health Profile, que foi dividido em DHP-1 e DHP-18, porquanto o DHP-1 (47) foi desenvolvido especificamente para DMl, com 32 questões em três domínios (barreiras para atividade, alimentação desinibida e perturbação psicológica), e o DHP-18 (48) que é uma adaptação do DHP-1 para ser utilizado em pacientes com DM2, com 18 itens, em razão da retirada de alguns itens que não se aplicavam na DM2. A organização dos domínios permaneceu a mesma. A consistência interna (alfa de Cronbach) variou entre 0,77 até 0,88 para o DHP- 1 e 0,71 até 0,88 para DHP-18 (42).

A Diabetes Impact Measurement Scales (DIMS) (49) foi desenvolvido na Universidade da Califórnia, com 44 questões, e abrange quatro domínios: sintomas específicos da DM, sintomas não-específicos, bem-estar, moral relacionada ao DM e desempenho do papel social. Pode ser utilizado em pacientes com DMl ou DM2. Possui consistência interna (alfa de Cronbach) variando entre 0,60 até 0,85 (42).

A Appraisal of Diabetes Scale (ADS) (50), questionário auto-administrado com 7 itens, pode ser utilizado tanto para o DMl quanto o DM2. Não há, até o momento, estudos sobre a adequação deste instrumento para pacientes idosos ou crianças (51). Detém consistência interna (alfa de Cronbach) de 0,73 (42).

O Audit of Diabetes-Dependent Quality of Life (ADDQoL) (52) é um questionário de 13 itens, avaliando a percepção do indivíduo sobre: funcionalidade física, bem-estar psicológico, bem-estar social, atividades funcionais e crescimento pessoal. Em cada questão é feito questionamento sobre como seria a vida do pesquisado (em cada domínio), se este não tivesse DM. Cada item utiliza escala, que varia de -3 a +3 , e o paciente deve indicar qual o peso que ele atribui à questão (de 0 a 3 ). Sua consistência interna (alfa de Cronbach) foi de $0,84(42)$.

O Questionnaire on Stress in Patients with DiabetesRevised (QSD-R) (53) é uma revisão do Questionnaire on Stress in Patients with Diabetes (QSD) (54). Esta versão revisada é mais curta e contém 45 questões - a versão original foi desenvolvida na Alemanha com 90 itens e possui consistência interna que oscila entre 0,63 até $0,88(55)$ - sobre estresse psicológico associado com problemas da vida diária em pacientes com DM. Com oito domínios (tempo de lazer, depressão ou medo do futuro, hipoglicemia, regime alimentar, reclamações físicas, trabalho, companheiro e relacionamento médico-paciente), cada item recebe pontuação que reflete a intensidade do problema ( 1 indica um pequeno problema e 5 aponta um problema muito grande). Observou-se consistência interna (alfa de Cronbach) variando entre 0,69 até 0,81 (42).

O Well-Being Enquiry for Diabetics (WED) (45) é composto de 50 questões, utilizando escala de 5 pontos, em quatro domínios (sintomas, desconforto, serenidade e impacto). O WED pode ser aplicado tanto para pacientes com DM tipo 1 quanto tipo 2. Possui consistência interna (alfa de Cronbach) variando entre 0,81 até $0,84(42)$.

O Diabetes 39 (D-39) (55) são 39 questões, abrangendo cinco dimensões (energia e mobilidade, controle do diabetes, ansiedade e preocupação, impacto social e funcionamento sexual). Pode ser utilizado em toda a população de pacientes com DM, independentemente das variáveis demográficas, sexo, idade, nível de educação, nível de saúde ou raça. A validade do instrumento foi avaliada para a população idosa. Foi relatada consistência interna (alfa de Cronbach) variando entre 0,81 até $0,93(42)$.

O Problem Areas in Diabetes (PAID) (56) é um questionário de 20 itens, focado nos aspectos emocionais negativos de o paciente viver com DM. Cada item pode ser pontuado de 1 (que significa "nenhum problema") até 6 (correspondendo a "problema sério"). O escore é transformado em uma escala que varia de 0 até 100; pontuação elevada indica maior prejuízo. Já existe versão traduzida para o português e validada para uso no Brasil (57). Demonstrou associação positiva com os níveis de hemoglobina glicada e como preditor de baixa aderência ao tratamento (58). Sua consistência interna (alfa de Cronbach) estudada foi de 0,95 (59).

Há também outras escalas mais específicas, como a escala para o uso de insulina (Diabetes Fear of Injecting and Self-Testing Questionnaire, Insulin Treatment Satisfaction Questionnaire e a Patient Satisfaction with Insulin Therapy Questionnaire), para crianças e jovens (Impact of Child Illness Scale e a Diabetes Quality of Life for Youth Scale), questões psicossociais (Diabetes Attitude Scale, Diabetes Knowledge Test, Diabetes Empowerment Scale e a Diabetes Family Behavior Scale) e complicações da DM (Diabetic Foot Ulcer Scale, Hypoglycemia Fear Survey, Michigan Neuropathy Screening Instrument e a Norfolk Quality of Life Questionnaire Diabetic Neuropathy) (60).

O Quadro 1 apresenta o resumo dos instrumentos específicos mais utilizados para DM. 
Quadro 1. Instrumentos específicos para utilização no diabetes melito e suas características.

\begin{tabular}{lccccc}
\hline Instrumento & DM1 & DM2 & $\begin{array}{c}\text { Alfa de } \\
\text { Cronbach }\end{array}$ & $\begin{array}{c}\text { Número } \\
\text { de itens }\end{array}$ & $\begin{array}{c}\text { Auto- } \\
\text { administrado }\end{array}$ \\
WED & $\sqrt{ }$ & $\sqrt{ }$ & $0,81-0,84$ & 50 & $\mathrm{Sim}$ \\
\hline D-39 & $\sqrt{ }$ & $\sqrt{ }$ & $0,81-0,93$ & 39 & $\mathrm{Sim}$ \\
PAID** & $\sqrt{ }$ & $\sqrt{ }$ & 0,95 & 20 & $\mathrm{Sim}$ \\
DCP & - & $\sqrt{ }$ & $0,60-0,95$ & 234 & $\mathrm{Sim}$ \\
DQOL & $\sqrt{ }$ & $\sqrt{ }$ & $0,67-0,92$ & 46 & $\mathrm{Sim}$ \\
DSQOLS & $\sqrt{ }$ & - & $0,70-0,88$ & 64 & $\mathrm{Sim}$ \\
DHP-1 & $\sqrt{ }$ & - & $0,77-0,88$ & 32 & $\mathrm{Sim}$ \\
\hline DHP-18 & - & $\sqrt{ }$ & $0,71-0,88$ & 18 & $\mathrm{Sim}$ \\
QSD-R & $\sqrt{ }$ & $\sqrt{ }$ & $0,69-0,81$ & 45 & $\mathrm{Sim}$ \\
ADDQOL* & $\sqrt{ }$ & $\sqrt{ }$ & 0,84 & 13 & $\mathrm{Sim}$ \\
DIMS & $\sqrt{ }$ & $\sqrt{ }$ & $0,60-0,85$ & 44 & $\mathrm{Sim}$ \\
ADS & $\sqrt{ }$ & $\sqrt{ }$ & 0,73 & 7 & $\mathrm{Sim}$ \\
\hline
\end{tabular}

$V$ instrumento que pode ser aplicado e que foi testado nestas populações: * = instrumentos com versão traduzida para língua portuguesa; ** = instrumentos com versão traduzida e validada para nosso meio.

\section{QUAL ESCALA ESCOLHER?}

Vantagens e desvantagens têm sido demonstradas tanto com a utilização de instrumentos genéricos quanto de específicos. A utilização de instrumentos mais específicos é defendida como forma de identificar as características relacionadas a determinado distúrbio (61). É questionada, no entanto, por ser abordagem restrita aos sintomas e às disfunções específicas, contribuindo pouco para visão mais ampla da QV (52). Outra limitação do uso de instrumentos específicos é a impossibilidade de comparação entre duas patologias distintas, por exemplo, diabetes com enfisema. Assim, não existe consenso sobre qual tipo de instrumento seria o melhor.

Em artigo de revisão sobre os instrumentos de avaliação de QV em pacientes com DM, Garratt, Schmidt e Fitzpatrick (5l) fazem uma crítica ao DQOL, ao WED e ao DIMS, questionando a falta de apoio empírico para dimensões que eles pretendiam medir. O DQOL e o DIMS têm evidência mais fraca para confiabilidade, enquanto o ADDQOL, o DHP-1/18, o DSQOLS, o D-39 e o QSD-R apresentam estimativas de confiabilidade aceitáveis para comparações clínicas de grupo.

Entre os instrumentos genéricos, os mais utilizados na literatura são o NHP, o SIP e o SF-36. O NHP e o SIP, no entanto, possuem respostas tendenciosas para saúde positiva em pacientes diabéticos, possivelmente em razão das limitações dos instrumentos, tornando-os inadequados para uso em DM (62).

O SF-36 é o instrumento genérico de avaliação de QV mais utilizado em pesquisas (63). A validade e a confiabilidade do SF-36 por meio de seus domínios são demonstradas em indivíduos com várias condições médicas, incluindo o DM (64). Observou-se consistência interna (alfa de Cronbach) acima de 0,80 para todos os oito domínios (65). Quando comparado com instrumentos específicos para DM, o SF-36 foi considerado também adequado para avaliar pacientes com DM (61). Outros investigadores, contudo, sugerem o uso de instrumentos específicos, associados ou isoladamente, para melhor avaliação desta população, por terem observado que a utilização isolada do SF-36 pode não demonstrar alteração na QV, a despeito da melhora clínica real $(66,67)$.

Considerando as diferenças entre as formas mais prevalentes de $\mathrm{DM}$, a não especificidade dos instrumentos para avaliar QV no DM é discutida, uma vez que, em sua maioria, os instrumentos foram desenhados para ter aplicação tanto em pacientes com $\mathrm{DMl}$ quanto 2 , com exceções do DHP-1/18, que tem versões específicas para cada tipo de DM, e dos DQOL e DSQOLS, específicos para pacientes com DMl.

Watkins e Connel (42) sugerem que o D-39, o DIMS, o QSD-R e o PAID sejam os instrumentos mais apropriados para o uso em qualquer população portadora de DM; o DCP e o DHP são mais relevantes em relação à DM2; enquanto isso, o DQOL, o DSQOLS e o WED são claramente direcionados para DMl.

No Brasil, o SF-36 é um dos poucos instrumentos que passou pelo processo formal de validação. Em 2006, Pagani e Pagani Jr. (68) verificaram, por meio de revisão das publicações dos últimos seis anos, utilizando as bases de dados Lilacs e SciELO, abordando a validação de questionários de QVRS para o Brasil, a existência de dez publicações, não sendo encontrado nenhum questionário específico para DM. Existem traduções para o português do PAID (61), do ADDQoL (61) e do DQOL (69), mas somente o PAID (60) e o DQOL (69) se encontram validados para uso em populações brasileiras. Outros instrumentos que também passaram pelo processo de validação foram o Diabetes Knowledge Scale (DKN-A) e o Diabetes Attitudes Questionnaires (ATT-19), específicos para avaliação do conhecimento e das atitudes de pacientes com DM e não de QV (70). Enquanto o DKN-A é um questionário que mede o conhecimento do paciente sobre DM, o ATT-19 avalia os aspectos psicológicos e emocionais 
sobre a DM. Sua principal aplicação foi associada à avaliação da intervenção educacional (70).

\section{CONSIDERAÇÕES FINAIS}

Em estudos longitudinais, o impacto psicossocial do DM está fortemente relacionado com a predição de mortalidade ou morbidade em pacientes com DM, inclusive sendo maior do que vários parâmetros clínicos e fisiológicos (impacto social da DM, idade, função renal, complexidade do regime dietético e história de fumo são os cinco maiores fatores associados com o risco de mortalidade) (71).

A utilização de instrumentos de avaliação de QVRS em pacientes com DM continua crescendo. Existem vários instrumentos com capacidade de aferir a $\mathrm{QV}$ em pacientes com DM, mas nenhum deles tem a capacidade claramente superior em relação ao outro. Em decorrência do elevado número de escalas específicas e genéricas utilizadas, há grande dificuldade em comparar a $\mathrm{QV}$ em pacientes diabéticos de grupos diferentes, principalmente quando considerados seus fatores determinantes. Os domínios utilizados e os parâmetros diferentes prejudicam a comparação de dois grupos de pacientes com DM, avaliados com escalas diferentes. Além disso, alguns instrumentos são específicos para determinados tipos de DM. Portanto, se faz necessária melhor compreensão do que determina a QVRS em pacientes com DM, considerando-se o grande número de variáveis que está provavelmente envolvida e que pode influenciar positiva ou negativamente a $\mathrm{QV}$.

A utilização de único instrumento para avaliação não está recomendada no presente estado de conhecimento. A análise prévia de qual população será estudada ajuda na escolha destes instrumentos. Outro ponto importante é a utilização de instrumentos validados para o nosso meio, pois a tradução isolada para o português não é suficiente para assegurar as propriedades psicométricas de determinado instrumento.

Assim sendo, o uso combinado do SF-36, instrumento genérico traduzido e validado para uso no Brasil e já amplamente utilizado em nosso meio, juntamente com o PAID, instrumento específico para DM que possui alto alfa de Cronbach $(0,95)$ e já foi traduzido e validado para uso no Brasil, parece ser a forma, neste momento, mais adequada de avaliar a $\mathrm{QV}$ em pacientes diabéticos no Brasil (34). O uso associado da versão brasileira do DQOL (DQOL-Brasil) como instrumen- to específico para o DM é outra alternativa válida (alfa de Cronbach $=0,92)(69)$.

Finalmente, a partir dos dados revistos no presente artigo, ressalta-se a necessidade urgente do desenvolvimento e da correta validação dos diversos instrumentos, sobretudo aqueles específicos, para uso em pacientes brasileiros com DM em contextos clínicos e de pesquisa.

Conflito de interesse: Não há conflito de interesse científico de nenhum dos autores.

\section{REFERÊNCIAS}

1. Ramos ATC, Crepaldi AL. Qualidade de vida em doenças pulmonares crônicas: aspectos conceituais e metodológicos. J Pneumologia. 2000;26:207-13.

2. Alberti KG, Zimmet PZ. Definition, diagnosis and classification of diabetes mellitus and its complications. Part 1: diagnosis and classification of diabetes mellitus provisional report of a WHO consultation. Diabet Med. 1998;15(7):539-53.

3. Selli L, Papaleo LK, Meneghel SN, Torneros JZ. Educational techniques in diabetes treatment. Cad Saude Publica. 2005;21(5):1366-72.

4. Favarato MECDS, Favarato D, Hueb WA, Aldrighi JM. Qualidade de vida em portadores de doença arterial coronária: comparação entre gêneros. Rev Assoc Med Bras. 2006;52:236-41.

5. Santos AMB, Assumpção A, Matsutani LA, Pereira CAB, Lage LV, Marques AP. Depressão e qualidade de vida em pacientes com fibromialgia. Rev Bras Fisioter. 2006;10:317-24.

6. WHO (World Health Organization). Constitution of the World Health Organization. Genebra: WHO; 1946.

7. Ciconelli RM. Tradução para o português e validação do questionário genérico de avaliação de qualidade de vida "Medical Outcomes Study 36-Item Short-Form Health Survey (SF-36)" [tese] São Paulo: Universidade Federal de São Paulo; 1997.

8. Efficace F, Bottomley A, Osoba D, Gotay C, Flechtner H, $\mathrm{d}^{\prime}$ Haese $\mathrm{S}$, et al. Beyond the development of Health-Related Quality-of-Life (HROOL) measures: a checklist for evaluating HROOL outcomes in cancer clinical trials - does HROOL evaluation in prostate cancer research inform clinical decision making? J Clin Oncol. 2003;21(18):3502-11.

9. Maia Filho HS, Gomes MM. Análise crítica dos instrumentos de avaliação da qualidade de vida na epilepsia infantil. J Epilepsy Clin Neurophysiol. 2004;10(3):147-53.

10. Wood-Dauphinee S. Assessing quality of life in clinical research: from where have we come and where are we going? J Clin Epidemiol. 1999;52(4):355-63.

11. Patrick DL, Bush JW, Chen MM. Toward an operational definition of health. J Health Soc Behav. 1973;14(1):6-23.

12. Patrick DL, Bush JW, Chen MM. Methods for measuring levels of well-being for a health status index. Health Serv Res. 1973;8(3):228-45.

13. Bergner M, Bobbitt RA, Pollard WE, Martin DP, Gilson BS. The sickness impact profile: validation of a health status measure. Med Care. 1976;14(1):57-67.

14. Gill TM, Feinstein AR. A critical appraisal of the quality of quality-of-life measurements. JAMA. 1994;272(8):619-26. 
15. Fleck MPA, Lousada S, Xavier M, Chachamovich E, Vieira G, Santos $L$, et al. Aplicação da versão em português do instrumento de avaliação de qualidade de vida da Organização Mundial da Saúde (WHOQOL-100). Rev Saude Publ. 1999;33: 198-205.

16. The WHOQOL Group. The World Health Organization quality of life assessment (WHOQOL): position paper from the World Health Organization. Soc Sci Med. 1995;10:1403-9.

17. Eiser C, Tooke JE. Quality-of-life evaluation in diabetes. Pharmacoeconomics. 1993;4(2):85-91.

18. Edelman D, Olsen MK, Dudley TK, Harris AC, Oddone EZ. Impact of diabetes screening on quality of life. Diabetes Care. 2002;25(6):1022-6.

19. Talley NJ, Young L, Bytzer P, Hammer J, Leemon M, Jones $M$, et al. Impact of chronic gastrointestinal symptoms in diabetes mellitus on health-related quality of life. Am J Gastroenterol. 2001;96(1):71-6.

20. Mata CM, Roset GM, Badia L, X, Antonanzas VF, Ragel AJ. Effect of type-2 diabetes mellitus on the quality of life of patients treated at primary care consultations in Spain. Aten Primaria. 2003;31(8):493-9.

21. Hill-Briggs F, Gary TL, Hill MN, Bone LR, Brancati FL. Healthrelated quality of life in urban African Americans with type 2 diabetes. J Gen Intern Med. 2002;17(6):412-9.

22. Beck-Nielsen H, Richelsen B, Schwartz SN, Hother NO. Insulin pump treatment: effect on glucose homeostasis, metabolites, hormones, insulin antibodies and quality of life. Diabetes Res. 1985;2(1):37-43.

23. Bott U, Ebrahim S, Hirschberger S, Skovlund SE. Effect of the rapid-acting insulin analogue insulin aspart on quality of life and treatment satisfaction in patients with type 1 diabetes. Diabet Med. 2003;20(8):626-34.

24. Pibernik-Okanovic M, Szabo S, Metelko Z. Quality of life following a change in therapy for diabetes mellitus. Pharmacoeconomics. 1998; 14(2):201-7.

25. Quality of life in type 2 diabetic patients is affected by complications but not by intensive policies to improve blood glucose or blood pressure control (UKPDS 37). U.K. Prospective Diabetes Study Group. Diabetes Care. 1999;22(7):1125-36.

26. Lloyd A, Sawyer W, Hopkinson P. Impact of long-term complications on quality of life in patients with type 2 diabetes not using insulin. Value Health. 2001;4(5):392-400.

27. Kolotkin RL, Crosby RD, Williams GR. Assessing weight-related quality of life in obese persons with type 2 diabetes. Diabetes Res Clin Pract. 2003;61(2):125-32.

28. Kaukua JK, Pekkarinen TA, Rissanen AM. Health-related quality of life in a randomised placebo-controlled trial of sibutramine in obese patients with type II diabetes. Int J Obes Relat Metab Disord. 2004;28(4):600-5.

29. Edgell ET, Coons SJ, Carter WB, Kallich JD, Mapes D, Damush $\mathrm{TM}$, et al. A review of health-related quality-of-life measures used in end-stage renal disease. Clin Ther. 1996;18(5):887-938.

30. Hays RD, Kallich JD, Mapes DL, Coons SJ, Carter WB. Development of the kidney disease quality of life (KDOOL) instrument. Qual Life Res. 1994;3(5):329-38.

31. Duarte PS, Miyazaki MCOS, Ciconelli RM, Sesso R. Tradução e adaptação cultural do instrumento de avaliação de qualidade de vida para pacientes renais crônicos (KDQOL-SF TM). Rev Assoc Med Bras. 2003;49(4):375-81.

32. Webb SM, Prieto L, Badia X, Albareda M, Catala M, Gaztambide $S$, et al. Acromegaly quality of life questionnaire (ACRO$\mathrm{QOL}$ ) a new health-related quality of life questionnaire for patients with acromegaly: development and psychometric properties. Clin Endocrinol (Oxf). 2002; 57(2):251-8.

33. Trepp R, Everts R, Stettler C, Fischli S, Allemann S, Webb SM, et al. Assessment of quality of life in patients with uncontrolled vs. controlled acromegaly using the Acromegaly Quality of Life Questionnaire (AcroQoL). Clin Endocrinol (Oxf). 2005;63(1): 103-10.

34. Duarte PS, Ciconelli RM. Instrumentos para a avaliação da qualidade de vida: genéricos e específicos. In: Schor N. Qualidade de vida. São Paulo: Manole; 2006.

35. EuroQol - a new facility for the measurement of health-related quality of life. The EuroOol group. Health Policy. 1990;16(3): 199-208.

36. Clarke P, Gray A, Holman R. Estimating utility values for health states of type 2 diabetic patients using the EQ-5D (UKPDS 62). Med Decis Making. 2002;22(4):340-9.

37. Kaplan RM, Bush JW, Berry CC. Health status: types of validity and the index of well-being. Health Serv Res. 1976;11(4): 478-507.

38. Kaplan RM, Ganiats TG, Sieber WJ. The quality of well-being scale, self-administered. Copyrighted material; 1996.

39. Ware Jr JE, Sherbourne CD. The MOS 36-item short-form health survey (SF-36). I. Conceptual framework and item selection. Med Care. 1992;30(6):473-83.

40. Dantas RA, Sawada NO, Malerbo MB. Research on quality of life: review of the scientific production of public universities from the state of Sao Paulo. Rev Lat Am Enfermagem. 2003;11(4):532-8.

41. Fitzgerald JT, Davis WK, Connell CM, Hess GE, Funnell MM, Hiss RG. Development and validation of the diabetes care profile. Eval Health Prof. 1996;19(2):208-30.

42. Watkins $\mathrm{K}$, Connell $\mathrm{CM}$. Measurement of health-related $\mathrm{QOL}$ in diabetes mellitus. Pharmacoeconomics. 2004;22(17):1109-26.

43. Reliability and validity of a diabetes quality-of-life measure for the diabetes control and complications trial (DCCT). The DCCT Research Group. Diabetes Care. 1988;11(9):725-32.

44. Eiser C, Flynn M, Green E, Havermans T, Kirby R, Sandeman D, et al. Quality of life in young adults with type 1 diabetes in relation to demographic and disease variables. Diabet Med. 1992;9(4):375-8.

45. Mannucci E, Ricca V, Bardini G, Rotella CM. Well-being enquiry for diabetics: a new measure of diabetes-related quality of life. Diab Nutr Metab. 1996;9(2):89-102.

46. Bott $\mathrm{U}$, Muhlhauser I, Overmann $\mathrm{H}$, Berger M. Validation of a diabetes-specific quality-of-life scale for patients with type 1 diabetes. Diabetes Care. 1998;21(5):757-69.

47. Meadows K, Steen N, McColl E, Eccles M, Shiels C, Hewison J, et al. The diabetes health profile (DHP): a new instrument for assessing the psychosocial profile of insulin requiring patients - development and psychometric evaluation. Qual Life Res. $1996 ; 5(2): 242-54$

48. Meadows KA, Abrams C, Sandbaek A. Adaptation of the diabetes health profile (DHP-1) for use with patients with type 2 diabetes mellitus: psychometric evaluation and cross-cultural comparison. Diabet Med. 2000;17(8):572-80.

49. Hammond GS, Aoki TT. Measurement of health status in diabetic patients. Diabetes impact measurement scales. Diabetes Care. 1992;15(4):469-77.

50. Carey MP, Jorgensen RS, Weinstock RS, Sprafkin RP, Lantinga LJ, Carnrike Jr CL, et al. Reliability and validity of the appraisal of diabetes scale. J Behav Med. 1991;14(1):43-51.

51. Garratt AM, Schmidt L, Fitzpatrick R. Patient-assessed health outcome measures for diabetes: a structured review. Diabet Med. 2002;19(1):1-11. 
52. Bradley C, Todd C, Gorton T, Symonds E, Martin A, Plowright $R$. The development of an individualized questionnaire measure of perceived impact of diabetes on quality of life: the ADDQoL. Qual Life Res. 1999;8(1-2):79-91.

53. Herschbach P, Duran G, Waadt S, Zettler A, Amm C, MartenMittag B. Psychometric properties of the questionnaire on stress in patients with diabetes - revised (QSD-R). Health Psychol. 1997;16(2):171-4.

54. Duran G, Herschbach P, Waadt S, Strian F, Zettler A. Assessing daily problems with diabetes: a subject-oriented approach to compliance. Psychol Rep. 1995;76(2):515-21.

55. Boyer JG, Earp JA. The development of an instrument for assessing the quality of life of people with diabetes. Diabetes-39. Med Care 1997;35(5):440-53.

56. Polonsky WH, Anderson BJ, Lohrer PA, Welch G, Jacobson AM, Aponte JE, et al. Assessment of diabetes-related distress. Diabetes Care. 1995;18(6):754-60.

57. Gross CC, Scain SF, Scheffel R, Gross JL, Hutz CS. Brazilian version of the problem areas in diabetes scale (B-PAID): validation and identification of individuals at high risk for emotional distress. Diabetes Res Clin Pract. 2007;76(3):455-9.

58. Snoek FJ, Pouwer F, Welch GW, Polonsky WH. Diabetes-related emotional distress in Dutch and U.S. diabetic patients: cross-cultural validity of the problem areas in diabetes scale. Diabetes Care. 2000;23(9):1305-9.

59. Welch GW, Jacobson AM, Polonsky WH. The problem areas in diabetes scale. An evaluation of its clinical utility. Diabetes Care. 1997;20(5):760-6.

60. Mapi Research Institute. QOLID The quality of life instruments database. 2007.

61. Nanda U, Andresen EM. Health-related quality of life. A guide for the health professional. Eval Health Prof. 1998;21(2):179-215.

62. Anderson RM, Fitzgerald JT, Wisdom K, Davis WK, Hiss RG. A comparison of global versus disease-specific quality-of-life measures in patients with NIDDM. Diabetes Care. 1997;20(3): 299-305.

63. Bardsley MJ, Astell S, McCallum A, Home PD. The performance of three measures of health status in an outpatient diabetes population. Diabet Med. 1993;10(7):619-26.
64. Hill-Briggs F, Gary TL, Baptiste-Roberts K, Brancati FL. Thirtysix-item short-form outcomes following a randomized controlled trial in type 2 diabetes. Diabetes Care. 2005;28(2):443-4.

65. Ware Jr JE, Gandek B. Methods for testing data quality, scaling assumptions, and reliability: the IQOLA Project approach. International quality of life assessment. J Clin Epidemiol. 1998;51(11):945-52.

66. Ware Jr JE, Kosinski M, Bayliss MS, McHorney CA, Rogers WH, Raczek A. Comparison of methods for the scoring and statistical analysis of SF-36 health profile and summary measures: summary of results from the Medical Outcomes Study. Med Care. 1995;33(4):264-79.

67. Hill-Briggs F, Gary TL, Baptiste-Roberts K, Brancati FL. Thirtysix-item short-form outcomes following a randomized controlled trial in type 2 diabetes. Diabetes Care. 2005;28(2):443-4.

68. Pagani TCS, Pagani Jr CR. Instrumentos de avaliação de qualidade de vida relacionada à saúde. Rev Ciências Bio. 2005;32-7.

69. Correr CJ, Patarolo R, Melchiors AC, Rossignoli P, FernandézLlimós F, Radominski RB. Tradução para o português e validação do diabetes quality of life measure (DQOL-Brasil). Arq Bras Endocrinol Metabol. 2008;52(3):515-22.

70. Torres HC, Virginia A, Schall VT. Validação dos questionários de conhecimento (DKN-A) e atitude (ATT-19) de diabetes mellitus. Rev Saude Publ. 2005;39:906-11.

71. Davis WK, Hess GE, Hiss RG. Psychosocial correlates of survival in diabetes. Diabetes Care. 1988;11(7):538-45.

\section{Endereço para correspondência:}

Renan Magalhães Montenegro Junior

Serviço de Endocrinologia e Diabetes do HUWC-FM-UFC

Rua Capitão Francisco Pedro, 1290

60430-370 Fortaleza, CE

E-mail: renanjr@ufc.br ou renanmontenegrojr@terra.com.br 